\title{
Comparative Study of Three $\beta$ Lactamase Test Methods in Staphylococcus aureus Isolated from Two Nepalese Hospitals
}

\author{
Shrestha Bidya1, Rana Shamser Suman² \\ ${ }^{1}$ Department of Microbiology, Tri-Chandra Campus, Tribhuvan University, Kathmandu, Nepal \\ ${ }^{2}$ Department of Medicine, Nepal Police Hospital, Kathmandu, Nepal \\ Email: $\underline{b}$ shrestha 07@hotmail.com
}

Received 13 January 2014; revised 13 February 2014; accepted 20 February 2014

Copyright (C) 2014 by authors and Scientific Research Publishing Inc.

This work is licensed under the Creative Commons Attribution International License (CC BY). http://creativecommons.org/licenses/by/4.0/

(c) (i) Open Access

\begin{abstract}
Background: $\beta$ lactamase is a plasmid-encoded enzyme that hydrolyzes $\beta$ lactam ring of $\beta$ lactam antibiotics rendering them ineffective. These enzymes, produced by Staphylococcus aureus along with many other organisms, have hindered the use of many useful and once life-saving $\beta$ lactam antibiotics from clinical practice. Methods: This study was aimed to compare three test methodschromogenic, acidimetric and iodometric-for the detection of $\beta$ lactamase enzyme produced by 404 nosocomial induced $S$. aureus isolated from two Nepali hospitals, Kathmandu based hospital (KBH) and Lalitpur based Hospital (LBH). The study was carried out following standard methodology during November 2007 to June 2009 in the Department of Microbiology, Institute of Medicine, Kathmandu, Nepal. Sensitivity, specificity, efficiency, positive predictive value, and negative predictive values of the tests were calculated taking penicillin resistance and sensitivity as the standard. Results: Chromogenic method was found to be the most sensitive (98.93\%) and efficient $(\mathbf{9 8 . 5 1} \%)$ test and had a high positive predictive value $(\mathbf{9 9 . 4 6 \% )}$. Sensitivity $(98.4 \%)$ and efficiency $(98.27 \%)$ of iodometric method was found to be comparable to chromogenic test; its specificity $(96.55 \%)$ and positive predictive value $(99.73 \%)$ were the highest among the 3 tests. Acidimetric test was the least sensitive $(97.33 \%)$ and efficient $(96.78 \%)$. Of note, the sensitivity and specificity of these test methods have been compromised due to the negativity of few penicillin resistant isolates and positivity of some penicillin sensitive isolates, respectively. Conclusion: Chromogenic method was found comparatively to be the best test method for the detection of $\beta$ lactamase production. However, in contrast to the other two test methods whose reagents can be locally and economically prepared, chromogenic test's use has been impeded by its cost and unavailability in the local Nepali market.
\end{abstract}

\section{Keywords}

$\beta$ Lactam; $\beta$ Lactamase; Nosocomial; S. aureus; MSSA; MRSA; Nepal 


\section{Introduction}

Before the introduction of penicillin in 1940, patients with Staphylococcus aureus infection had a mortality of $90 \%$. With the advent of the penicillin era, remarkable improvement was observed in the recovery and survival of infected patients [1]. This historic triumph however was relatively short-lived—not too long after the introduction of penicillin in clinical use. S. aureus developed resistance to penicillin, making it the first microbe to challenge the sweeping efficacy of penicillin. By liberating $\beta$ lactamase enzyme, $S$. aureus acquired the ability to inactivate $\beta$ lactam ring of $\beta$ lactam antibiotics. Within a few years, most hospital isolates had become resistant to penicillin and were found in patients who were previously treated with the drug [2]. Till the early 1950s, penicillinase ( $\beta$ lactamase enzyme) producing $S$. aureus strains were universally present in hospitals, whereas community isolates continued to depict susceptibility. Then, a study conducted during 1957-1966 in Copenhagen showed that $85 \%$ - $90 \%$ of hospital strains and $65 \%-70 \%$ of community strains were resistant to penicillin, demonstrating that resistance was an increasingly alarming phenomenon and a much more prevalent conundrum than what had been originally anticipated [3]. Methicillin, a semi-synthetic derivative of benzyl penicillin was then used as a therapeutic agent in 1959 and 1960. But almost immediately upon its introduction in clinical practice in 1961, S. aureus acquired resistance to methicillin as well [4] — this time, instead of producing another round of $\beta$ lactamase enzyme [5] [6], S. aureus had learned to change the configuration of penicillin binding protein PBP, the very target protein of penicillin, thus rendering the drug hapless against this ever evolving bug.

The $\beta$ lactamase enzyme produced by $S$. aureus to gain resistance against $\beta$ lactam antibiotics like penicillin [7] is encoded in the bla gene, situated in the plasmid. This staphylococcal $\beta$ lactamase gene has promoter and regulatory regions, which are similar to those present in the mecA gene coding for methicillin resistance in methicillin resistant Staphylococcus aureus (MRSA) [8]. Within the regulatory region of bla gene, there are blaI and blaR1genes, which regulate $\beta$ lactamase production in S. aureus [9]. blaI and blaR1 encode a repressor and a signal transducer with antirepressor activity respectively [10]. blaI codes for BlaI repressor protein, which is a DNA binding protein that represses $\beta$ lactamase gene transcription. blaR1 on the other hand, codes for BlaR1, which is a signal transducing PBP that in the presence of $\beta$ lactam antibiotics, leads to $\beta$ lactamase gene transcription [11].

$\beta$ lactamase production can be detected by three different methods. Chromogenic method is based on the principle that hydrolysis of certain $\beta$ lactam antibiotic leads to a distinct color change from a light yellow to a deep red color. Acidimetric method uses a $\mathrm{pH}$ indicator color change from purple pink to yellow to detect the formation of at least one extra carboxyl group produced during the hydrolysis of $\beta$ lactam antibiotic by $\beta$ lactamase. And finally, the iodometric method detects the loss of blue color from a blue starch/iodine complex caused by the removal of iodine from the complex by the reducing action of a $\beta$ lactamase hydrolysis product. In this research study, $\beta$ lactamase production was studied in induced $S$. aureus isolates-both methicillin sensitive S. aureus (MSSA) and MRSA-and sensitivity, specificity, efficiency, and positive and negative predictive values of the three test methods used for its detection were compared.

\section{Material and Methods}

Consecutive clinical samples from patients with nosocomial infections in two hospitals, Kathmandu based hospital (KBH) and Lalitpur based hospital (LBH), were cultured and characterized in the Department of Microbiology, Institute of Medicine Kathmandu, Nepal, following standard methodology [12]. The samples included pus, wound swab, bed sore swab, tracheal aspirate, bronchial aspirate, sputum, urine, and high vaginal swab. Antibiotic susceptibility of the isolates was examined by following clinical laboratory standard institute (CLSI) 2007 protocol [13], using penicillin, oxacillin, cefoxitin along with many other non $\beta$ lactam antibiotics discs. A total of 404 S. aureus $(\mathrm{KBH}, \mathrm{n}=304$; LBH, $\mathrm{n}=100$ ) was isolated. In all isolates, irrespective of their susceptibility or resistance to penicillin, tests for $\beta$ lactamase production were performed by chromogenic, iodometric and acidimetric test methods. Induced microorganisms from the margin of penicillin zone of inhibition on Mueller Hinton agar (MHA) plate (antibiotic susceptibility test) were further studied. S. aureus isolates susceptible to cefoxitin and oxacillin were regarded as MSSA and resistant ones as MRSA. Oxacillin resistance was confirmed by oxacillin E test.

\section{Tests for $\beta$ Lactamase Production}

$\beta$ lactamase enzyme production was detected in all isolates as per the American Society for Microbiology (ASM) 
2004 [14] protocol. The tests were run in parallel with the reference strain S. aureus ATCC 25923.

Chromogenic method: Following instructions provided by the manufacturer, several colonies of the test organism were applied directly over a sterile, distilled, water-moistened nitrocefin disc [15]. A change in color from light yellow to deep red color within 15 seconds to 5 minutes was identified as hydrolysis of $\beta$ lactam antibiotic by induced $\beta$ lactamase enzyme. No change in color within 5 minutes was noted as negative.

Acidimetric method: Test organisms were suspended in penicillin-phenol red test reagent (prepared in house) dispensed in $100 \mu \mathrm{l}$ volume in mitrotitre wells. Change in color from purple pink to yellow within 15 minutes was regarded as positive and no change within 15 minutes was taken as negative for the production of $\beta$ lactamase [14].

Iodometric method: Penicillin solution (prepared in house) in $100 \mu$ l volume was dispensed in microtitre wells and test organisms were suspended in wells to a concentration of $10^{9}$ cells $/ \mathrm{ml}$ (McFarland's number 4). Two drops of $1 \%$ freshly prepared starch indicator were added in each well and thoroughly mixed, following which the tray was incubated at room temperature for 30 - 60 minutes. One drop of iodine reagent was dispensed in each well. Rapid decolorization of blue color within 10 minutes was regarded as positive for $\beta$ lactamase activity and persistence of blue color as negative [14].

Sensitivity, specificity, efficiency, and positive and negative predicative values were calculated as per Forbes et al., 2007 [16]. Penicillin resistance and sensitivity were taken as standard in all tests for calculation.

\section{Result}

Of 404 nosocomial S. aureus isolates, 92.8\% (375) were found to be resistant to penicillin and only $7.2 \%$ (29) were found to be sensitive 93.8\% (285) penicillin resistant isolates from KBH and 90\% (90) from LBH. All penicillin sensitive isolates were characterized to be MSSA. Positivity to all three $\beta$ lactamase tests by penicillin resistant isolates and negativity to these tests by penicillin sensitive isolates were not scored as expected (Table 1). Sensitivity, specificity, efficiency, positive predictive value, and negative predictive value of all testmethods are shown in Table 2. Of the three test methods, chromogenic test was the most sensitive and efficient and acidimetric test was the least so. Iodometric test was found to be the most specific test with sensitivity and efficiency comparable to those of the chromogenic method.

With the exception of 17 isolates, a uniform pattern emerged with all penicillin resistant isolates being positive and all penicillin sensitive isolates being negative in each of the 3 tests. Of the 17 isolates that departed from the norm, 12 were resistant to penicillin and 5 were sensitive. The 12 penicillin resistant isolates that gave incongruent results included MSSA, heterogeneous MRSA, and homogeneous MRSA. From this group of 12 iso-

Table 1. Result of $\beta$ lactamase tests of penicillin resistant and sensitive isolates.

\begin{tabular}{ccccc}
\hline \multirow{2}{*}{ Tests } & \multicolumn{2}{c}{ Penicillin resistant $(\mathbf{n}=\mathbf{3 7 5 )}$} & \multicolumn{2}{c}{ Penicillin sensitive (n= 29) } \\
\cline { 2 - 4 } & Negative, Number (\%) & Positive, Number (\%) & Positive, Number (\%) & Negative, Number (\%) \\
\hline Chromogenic & $4(1.1 \%)$ & $371(98.9 \%)$ & $2(6.9 \%)$ & $27(93.1 \%)$ \\
Iodometric & $6(1.6 \%)$ & $369(98.4 \%)$ & $1(3.4 \%)$ & $28(96.6 \%)$ \\
Acidimetric & $10(2.71 \%)$ & $365(97.3 \%)$ & $3(10.3 \%)$ & $26(89.7 \%)$ \\
\hline
\end{tabular}

Table 2. Sensitivity, specificity, positive and negative predictive values $\beta$ lactamase tests.

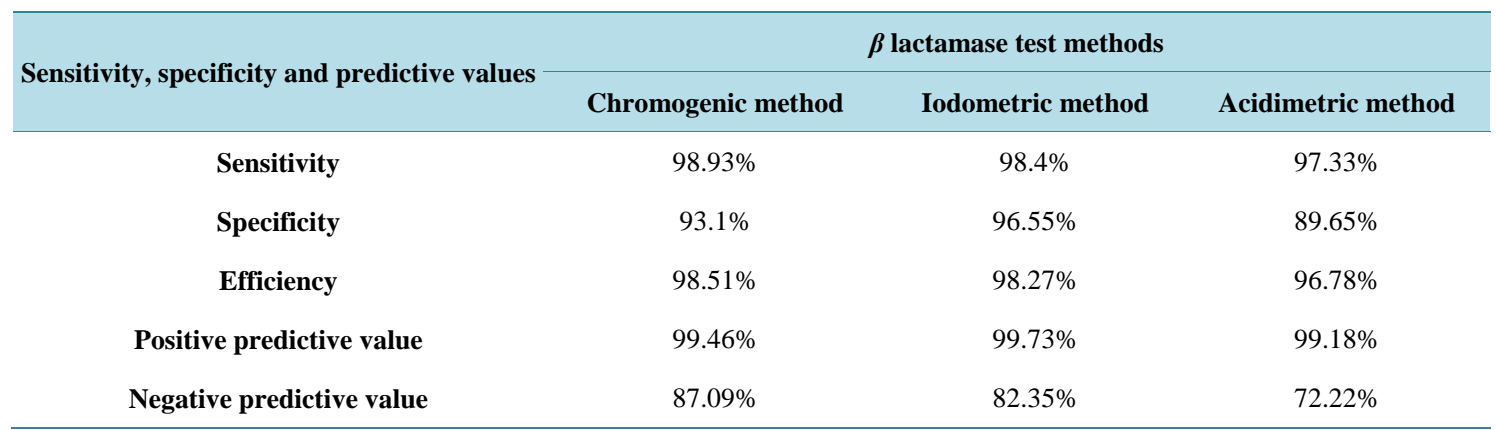


lates, 2 heterogeneous MRSA from KBH and 2 MSSA from LBH that were resistant to penicillin were negative in all of the 3 tests. Of the remaining 8 isolates, variable results to these tests were observed. Except for 2 isolates, positivity of chromogenic and iodometric tests were similar. However, resistance to penicillin 4, 6 and 10 isolates were negative (false negative) in chromogenic, iodometric and acidimetric test methods, respectively. It is important to note that due to these false negative results, sensitivity, efficiency, and negative predictive value of these $3 \beta$ lactamase tests compared to microbiological test have been compromised.

Five penicillin sensitive isolates that were MSSA from KBH gave variable results as well. Penicillin sensitive isolates 1, 2, and 3 produced false positive results in iodometric, chromogenic and acidimetric tests respectively thus decreasing the specificity and positive predictive value of these tests.

\section{Calculation of Sensitivity, Specificity, Positive and Negative Predictive Values of Three $\beta$ Lactamase Tests}

2 heterogeneous MRSA and 2 MSSA isolates that were penicillin resistant and negative in all 3 tests, penicillin resistant isolates that were negative in some of these tests, and penicillin sensitive isolates that were positive in some of these tests were included in the calculations for sensitivity, specificity, efficiency, and positive and negative predictive values.

\section{Discussion and Conclusion}

The present finding of the very high occurrence 92.8\% (93.8\% in KBH and 90\% in LBH) of $\beta$ lactamase producing nosocomial S. aureus isolates is higher than the reported $75 \%$ of $\beta$ lactamase production among hospital isolates from a Nepali Hospital in 2007 [17]. The difference is perhaps due to the fact that nosocomial isolates (present study group) are highly resistant to many antibiotics compared to hospital isolates.

It has been reported that rapid $\beta$ lactamase tests that include iodometric, acidimetric and chromogenic methods can yield clinically relevant information earlier than disk diffusion or minimum inhibitory concentration test [18]. To add to the utility of these $\beta$ lactamase tests, no significant difference has been reported between acidimetric/iodometric tests and microbiological cloverleaf test in a comparative study in India [19]. Of note, the sensitivity and specificity of these 3 biochemical test methods obtained in the present study are slightly lower than what has been reported so far. However, it has been stated that all three biochemical methods can be used for the detection of $\beta$ lactamase enzyme from S. aureus [14] [20]. We could have arrived at a similar conclusion from the current study had the 4 (2 LBH MSSA and 2 KBH heterogeneous MRSA) isolates resistant to penicillin not tested negative and had certain penicillin sensitive isolates not tested positive. In such a context, sensitivity, negative predictive value, specificity, and positive predictive value would not have been compromised.

What conferred negativity in $\beta$ lactamase test in penicillin resistant isolates is unknown as of yet, particularly since the present study utilized induced cells that have been reported to have greater sensitivity than non-induced cells [21]; the explanation would require genotyping for the presence of bla gene, which is beyond the aim of this research. Alternatively, Phoenix ${ }^{\text {TM }}$ Automated ID/AST system which as reported by Yu et al. in 1999 [21] can detect $\beta$ lactamase in strains that give variable cefinase (discs impregnated with nitrocefin) results, might be able to detect $\beta$ lactamase in these negative testing penicillin resistant isolates.

With high sensitivity, efficiency, and negative predictive value compared to acidimetric and iodometric methods, chromogenic method was found to be the best method for the detection of $\beta$-lactamase. In concordance to this finding, a high degree of efficacy for the chromogenic method on induced strains grown on antibiotic susceptibility agar and for starch iodine plate method has been reported [22]. Nitrocefin has been proved to be sensitive to hydrolysis by all known lactamases produced by Gram-positive and Gram-negative bacteria [23]. Four penicillin resistant isolates that were negative by all three $\beta$ lactamase tests have affected the sensitivity and specificity of these test methods. Had these isolates been positive, the sensitivity of chromogenic method would have approached 99\% with similar results for the other test methods as well. However, chromogenic method has been reported to be $100 \%$ correlated with resistance to penicillin and ampicillin [24] and to be the most specific test and perhaps the simplest method in use. Unlike other tests that have been known to give misleading results owing to the antibacterial activity of the hydrolysis product in microbiological method or interference by other chemical groups in biochemical methods, chromogenic method does not have any interference [25]. Chromogenic and microbiological techniques are sensitive to all types of $\beta$ lactamase activity and moreover, the present study, in agreement with various previous studies, has shown that the chromogenic test method is quicker and 
more convenient than the other two $\beta$ lactamase detection tests. One potential drawback of note is the limited availability of nitrocefin disc hinders its use.

With the exception of its negative predictive value, iodometric method also had sensitivity, specificity, efficiency, and positive predictive value comparable to the chromogenic method. Iodometric test is an easy-to-perform, economical, and relatively rapid method. Iodometric slide test results have been reported to be similar to nitrocefin disk test, and thus can be used in detecting staphylococcal $\beta$ lactamase when chromogenic methods are not available [26]. The present finding of greater sensitivity of the iodometric method compared to acidimetricis supported by reports that tube iodometric tests (used in the current study) are more accurate [27] and more sensitive that acidimetric methods [26] for the detection of $\beta$ lactamase production by staphylococci. However, it is important to note that acidimetric method has been reported to be more effective than iodometric test for the detection of coagulase positive staphylococcal $\beta$ lactamase [26]. Like the chromogenic method, iodometric test is specific to $\beta$ lactamase but is not as sensitive as the chromogenic method for the detection of enzymes from cephalosporinase activity. Further, difficulty in acquisition of freshly prepared starch and iodine solutions may hinder its routine use.

Acidimetric and microbiological techniques do not differentiate between acylase and $\beta$ lactamase activity. Despite being easy to read and interpret like its chromogenic counterpart, acidimetric technique is less sensitive for enzymes with cephalosporinase activity, quite like the iodometric test.

In iodometric and acidimetric tests, penicillin is used as the substrate and, therefore, the tests are equipped to detectpenicillinases. The higher sensitivity and efficiency of chromogenic method compared to thesetwo tests could be due to the detection of both penicillinase and cephalosporinase enzymes produced by the test isolatesin the chromogenic test. Nitrocefin disc utilized in the chromogenic method has been reported to be effective in detecting all known $\beta$ lactamases, including the staphylococcal penicillinases along with other microorganisms like Neisseria gonorrhoeae, Haemophilus influenza and Moraxella (Branhamella) catarrhalis [24] [28] [29]. It has been stated that iodometric method is suitable for testing $\beta$ lactamase production by $N$. gonorrhoeae, acidimetric method for Haemophilus spp., $N$. gonorrhoeae, and staphylococci, and nitrocefin is the only reliable test for detecting $\beta$ lactamase production by Enterococcus spp. [29].

Each of the detection methods may lead to misleading results due to the antibacterial activity of the hydrolysis product or interference by other chemical groups [25]. It is likely that the factors affecting nitrocefin detection method are less of a problem in these cases. Furthermore, its high sensitivity, specificity, easy-to-read and rapid result with lack of need for preparation of the reagent makes it a good choice for $\beta$ lactamase activity detection. However, nitrocefin should not entirely replace conventional susceptibility testing because other factors also influence the results of such tests, and on occasion, intrinsic resistance to $\beta$ lactam antimicrobials has not been correlated with the production of $\beta$ lactamase [18].

$\beta$ lactamase tests can predict the outcome of susceptibility tests with $\beta$ lactam antimicrobials. This study found the chromogenic test to be the most sensitive and efficient, with the iodometric test producing results comparable to the chromogenic test. Chromogenic test therefore is not only an easy-to-read, rapid test, but also a highly sensitive method that gives relatively fewer misleading results owing to hydrolysis products, and can detect all known $\beta$ lactamases, including staphylococcal penicillinase. Therefore, this test should be employed for the detection of staphylococcal $\beta$ lactamases, provided it is available in the local market.

\section{References}

[1] Daum, R.S. (1988) Community Acquired Methicillin Resistant Staphylococcal Infections. The Pediatric Infectious Disease Journal, 17, 745-746. http://dx.doi.org/10.1097/00006454-199808000-00015

[2] Barber, M. and Rozwadowska-Dowzenko, M. (1948) Infection by Penicillin Resistant Staphylococci. Lancet, 1, 641644. http://dx.doi.org/10.1016/S0140-6736(48)92166-7

[3] Jassen, O., Rosendel, K., Bulow, P., Faber, V. and Erikson, K.R. (1969) Changing Staphylococci and Staphylococcal Infections: A Ten Year Study of Bacteria and Cases of Bacterimia. The New England Journal of Medicine, 281, 627635. http://dx.doi.org/10.1056/NEJM196909182811201

[4] Jevons, M.P. (1961) “Celbenin”-Resistant Staphylococci. British Medical Journal, 1, 124-125. http://dx.doi.org/10.1136/bmj.1.5219.124-a http://www.ncbi.nlm.nih.gov/pmc/articles/PMC1952888/

[5] Hartman, B.S. and Tomasz, A. (1984). A Low Affinity Penicillin Binding Protein Associated in Beta Lactam Resistance in Staphylococcus aureus. Journal of Bacteriology, 158, 513-516. 
[6] Hackbarth, C.J. and Chambers, H.F. (1989) Methicillin Resistant Staphylococci: Detection Methods and Treatment of Infections. Antimicrob Agents and Chemother, 33, 995-999. http://dx.doi.org/10.1128/AAC.33.7.995

[7] Hugo, W.B. and Russell, A.D. (1986) Pharmaceutical Microbiology. 3rd Edition, Blackwell Scientific Publications, London.

[8] Song, M.D., Wachi, M., Doi, M., Ishino, F. and Matsuhashi, M. (1987) Evolution of an Inducible Penicillin—Target Protein in Methicillin Resistant Staphylococcus aureus by Gene Fusion. FEBS Letters, 221, 167-171. http://dx.doi.org/10.1016/0014-5793(87)80373-3

[9] Hiramatsu, K. (1995) Molecular Evolution of MRSA. Microbiology and Immunology, 39, 531-543. http://dx.doi.org/10.1111/j.1348-0421.1995.tb02239.x

[10] Joris, B., Hardt, K. and Ghuysen, G.M. (1994) Induction of $\beta$ Lactamase and Low Affinity Penicillin Binding Protein $2^{\prime}$ Synthesis in Gram Positive Bacteria. In: Ghuysen, J.M. and Hakenbeck, R.M., Eds., Bacterial Cell Wall, Elsevier Science, New York, 505-515.

[11] Chambers, H.F. (1997) Methicillin Resistance in Staphylococci: Molecular and Biochemical Basis and Clinical Implications. Clinical Microbiology Reviews, 10, 781-791.

[12] Isenberg, H.D. (2004) Clinical Microbiology Procedure Handbook. Vol. 1, American Society for Microbiology, ASM Press.

[13] Clinical Laboratory Standards Institute (2007) Performance Standard for Antimicrobial Susceptibility Testing: Seventeenth Informational Supplement M100-S17, Vol. 27, No. 1, Clinical Laboratory Standards Institute, Wayne.

[14] Isenberg, H.D. (2004) Clinical Microbiology Procedure Handbook. Vol. 2, American Society for Microbiology, ASM Press.

[15] Package Insert. Nitrocefin disc, BBL, USA.

[16] Forbes, B.A., Sahm, D.F. and Weissfeld, A. (2007) Bailey and Scott’s Diagnostic Microbiology. 12th Edition, Mosby Elsevier, St Louis, Missouri.

[17] Shrestha, B. (2007) Study of $\beta$ Lactamase Activity of Staphylococcus aureus Isolated from Healthy Nasal Carriers and Hospital Isolates. Nepal Medical College Journal, 9, 107-110.

[18] Richmond, M.H. (1979) Beta-Lactam Antibiotics and Beta-Lactamases: Two Sides of a Continuing Story. Clinical Infectious Diseases, 1, 30-38. http://dx.doi.org/10.1093/clinids/1.1.30

[19] Samant, S.A. and PAI, C. (2012) Comparative Evaluation of $\beta$ Lactamase Detection Methods in Staphylococci. International Journal of Research in Pharmaceutical and Biomedical Sciences, 3, 1580-1588.

[20] Oxoid. Nitrocefin (Glaxo Research 87/312). http://www.oxoid.com/UK/blue/index.asp?c=UK\&lang=EN

[21] Yu, C., Karr, T., Turner, D., Towns, V. and Sinha, J. (1999) Rapid Detection of Beta-Lactamase Production in Penicillin Sensitive Staphylococci by the Phoenix ${ }^{\mathrm{TM}}$ Automated ID/AST System. The European Congress of Clinical Microbiology and Infectious Disease (ECCMID), March 1999, Berlin.

[22] Petersson, A.C., Eliasson, I., Kamme, C. and Miorner, H. (1989) Evaluation of Four Qualitative Methods for Detection of Beta Lactamase Production in Staphylococcus aureus and Micrococcus Species. European Journal of Clinical Microbiology and Infectious Diseases, 8, 962-967. http://dx.doi.org/10.1007/BF01967566

[23] Nitrocefin|CAS 41906-86-9|Calbiochem | 5MG. http://www.merckmillipore.com

[24] Montgomery, K., Raymundo, L. and Drew, W.L. (1979) Chromogenic Cephalosporin Spot Test to Detect Beta-Lactamase in Clinically Significant Bacteria. Journal of Clinical Microbiology, 9, 205-207.

[25] Plested, S.J., Simpson, I.N. and James, M. (1983) The Detection of Bacterial $\beta$ Lactamase and Their Identification by Analytical Isoelectric Focusing. In: Russel, A.D. and Quesnel, L.B., Eds., Antibiotics: Assessment of Antimicrobial Activity and Resistance, Academic Press Inc., London.

[26] Kilic, E. and Cirak, Y. (2006) Comparison of Staphylococcal Beta Lactamase Detection Methods. Fabad Journal of Pharmaceutical Sciences, 31, 79-84.

[27] Devapriya, F., Ramesh, R., Khan, A.K.S. and Shanmugam, J. (2013) $\beta$-Lactamase Production of Staphylococcus Aureus: A Comparison Study of Different Iodometric Methods. Gulf Medical Journal, 2, 16-21.

[28] Sykes, R.B. and Matthew, M. (1976) The Beta-Lactamases of Gram-Negative Bacteria and Their Role in Resistance to Beta Lactam Antibiotics. Journal of Antimicrobial Chemotherapy, 2, 115-157. http://dx.doi.org/10.1093/jac/2.2.115

[29] Clinical and Laboratory Standards Institute (2006) Methods for Dilution Antimicrobial Susceptibility Test for Bacteria that Grow Aerobically. M7. Clinical and Laboratory Standards Institute, Wayne. 